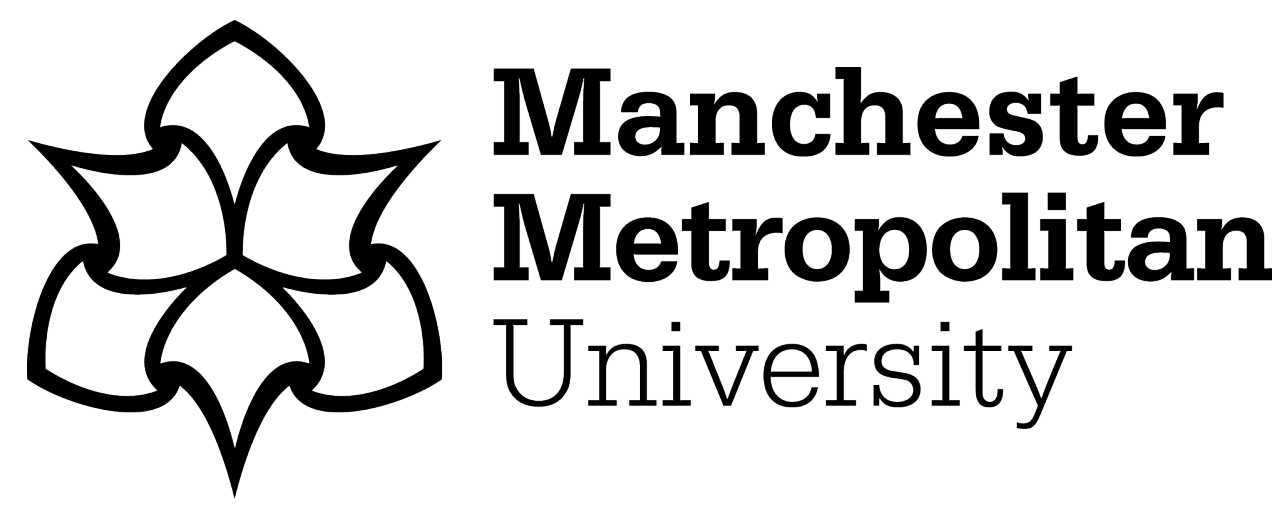

Kuithan, Pauline, Rushton, A, Sanderson, Andrew ORCID logoORCID: https://orcid.org/0000-0002-7892-1067, Heneghan, NR and Falla, D (2019) Lack of exercise induced hypoalgesia in response to repeated lifting in individuals with low back pain. In: Physiotherapy UK, 19 October 2018 - 20 October 2018, Birmingham, UK.

Downloaded from: https://e-space.mmu.ac.uk/627669/

Version: Accepted Version

Publisher: Elsevier

DOI: https://doi.org/10.1016/j.physio.2018.11.185

Usage rights: Creative Commons: Attribution-Noncommercial-No Derivative Works 4.0

Please cite the published version 


\title{
Lack of exercise induced hypoalgesia in response to repeated lifting in individuals with low back pain
}

\author{
P. Kuithan; A. Rushton; A. Sanderson; N.R. Heneghan; D. Falla
}

Purpose: Central and peripheral sensitisation is a major contributor to ongoing disability in chronic low back pain (LBP). Exercise as a management option is recommended in all clinical guidelines. In addition to its general effects, exercise induced hypoalgesia $(\mathrm{EIH})$, referring to a temporary change in pain sensitivity, is a documented effect. Few studies to date have investigated $\mathrm{EIH}$ in patients with LBP, with some evidence for impaired EIH potentially driven by peripheral and central sensitisation. The aim of the study was to investigate if pressure pain thresholds (PPT), as a measure of $\mathrm{EIH}$, are decreased in patients with LBP compared to healthy controls over the lumbar erector spinae and at a remote area after a prolonged lifting task.

Methods: An observational, case control study recruited participants with chronic LBP (>3 months) and healthy controls with ethical approval from the University of Birmingham. Psychometric data and the Oswestry disability index (ODI) characterised the participants. PPT measurements were taken over 8 sites at each side of the lower back and a remote site before and after the lifting task. To mimic an occupational task, participants were asked to lift a 5-kg box in a rotational pattern onto different shelves for $\sim 7$ minutes. Pain (NRS 0-10) and perceived exertion (Borg Scale (BS)) were recorded. Data were analysed using a three-way analysis of variance (ANOVA) with time, location and group as factors. ANOVA was followed by Student Newman Keuls (SNK) post hoc analysis.

Results: The sample comprised participants with chronic but relatively mild LBP (ODI = $16.0 \pm 7.0)(n=20)(9$ women, age $30.1 \pm 11.5)$ and healthy controls $(n=18)(11$ women, age $28.2 \pm 12.5)$. The LBP group perceived the task as significantly more fatiguing $(B S=13.1 \pm 1.7)$ and reported higher pain $(4.7 / 10 \pm 2.2)$ during lifting. PPT was dependent on group $(F=$ $35.1, p<0.00001)$, test location $(F=2.9, p<0.001)$, and the interaction between group and time $(F=9.2, p<0.01)$. Post hoc analysis revealed that the control subjects displayed higher PPTs across all locations both preand post- the repeated lifting task. However, unlike the control subjects which showed a significant increase in the PPT across all locations after the lifting task (SNK: $p<0.01$; pre: $341.4 \pm 116.9 \mathrm{kPa}$, post: $371.2 \pm 130.4$ $\mathrm{kPa})$, the participants with LBP did not display a significant change in the PPT pre- to post-testing (SNK: $p=0.15$; pre: $320.1 \pm 113.7 \mathrm{kPa}$, post: $305.2 \pm 101.0 \mathrm{kPa}$ ). No changes at remote sites could be detected. 
Conclusion(s): Asymptomatic people responded favourably to a repeated lifting task displaying evidence of EIH as revealed by a significant reduction in pressure pain sensitivity across the lumbar region. In contrast, this phenomenon was absent in participants with LBP. These findings have important implications for exercise prescription.

Implications: ElH was absent in individuals with relatively mild chronic LBP. Additional research is required to further elucidate the mechanisms and evaluate the effect of difference exercise interventions.

Funding acknowledgements: This work was unfunded. 